\title{
Der Internationale Mathematik Kongreß 1998 in Berlin - ein erster Bericht
}

\section{von Martin Aigner}

Am 30. Juli 1994 war es soweit: Die Generalversammlung der Internationalen Mathematischen Union (IMU) hatte Berlin einstimmig zum Tagungsort des nächsten Kongresses 1998 gewählt. Als erste Vorschau auf den ICM 98 möchte ich in den folgenden Zeilen berichten, was sich seither getan hat und was vom Berliner Kongreß zu erwarten ist.

\section{Ein Blick zurück}

Deutschland war schon einmal Gastgeber für den Internationalen Kongreß. Nach Zürich 1897 und Paris 1900 fand der Kongreß 1904 in Heidelberg mit etwa 300 Teilnehmern unter dem Vorsitz von Heinrich Weber statt. Nach dem Krieg gab es in den sechziger Jahren den ersten Versuch, den Kongreß wieder nach Deutschland zu holen. Herr Hirzebruch war als DMVVorsitzender in Halle 1961 beauftragt worden, eine Bewerbung für 1966 vorzubereiten. Dann kam beim ICM 1962 in Stockholm die Kandidatur aus Moskau. Da der ICM noch nie in einem sozialistischem Land stattgefunden hatte, wurde eine Bewerbung aus Deutschland nicht weiter verfolgt. Der nächste Anlauf war 1978 in Helsinki. Die DMV hatte zusammen mit der Universität Hamburg eine Bewerbung für 1982 vorbereitet. Bei der entscheidenden Sitzung erhielt jedoch Warschau den Zuschlag. Der Kongreß mußte schließlich wegen des Kriegsrechts in Polen um ein Jahr verschoben werden. Für den Kongreß 1990 hatten die Münchner Universitäten eine ausgereifte Bewerbung mit Finanzierungsplan eingereicht (von dem die Berliner Vorbereitungen in hohem Maße profitierten). Schließlich fiel die Wahl auf Kyoto, vor allem deshalb, weil noch nie ein ICM in Japan stattgefunden hatte. Die Idee, den Kongreß nach Berlin zu holen, wurde erstmals bei der DMV-Tagung 1987 in Berlin vorgestellt. Bei der Eröffnungsveranstaltung wurde (unter den skeptischen Blicken der anwesenden Politiker) der Wunsch geäußert, den Kongreß in beiden Teilen Berlins stattfinden zu lassen - mit freiem Transit zwischen den beiden Stadthälften. Wahrscheinlich eine Utopie unter den gegebenen Umständen, aber der Schwung reichte aus, um nach den politischen Veränderungen die Utopie Wirklichkeit werden zu lassen.

\section{Organisation des Kongresses}

Das engere Organisationskomitee wurde vom Präsidium der DMV gewählt mit Herrn Hirzebruch als Ehrenvorsitzenden und den Berliner Kollegen Grötschel (Konrad-Zuse- Zentrum und TU Berlin) als Vorsitzenden sowie den Kollegen Aigner (FU Berlin), Sprekels (Weierstraß-Institut und HU Berlin) und Wink- ler (TU Berlin). Zusätzlich wurden mehrere Unterkomitees ins Leben gerufen, die für Finanzierung, Stipendienangelegenheiten, Tagungen rund um den ICM 98, sowie für Öffentlichkeitsarbeit und das Rahmenprogramm verantwortlich sind.

\section{Wissenschaftliches Programm}

Das wissenschaftliche Programm und insbesondere die Auswahl der Sektionen (etwa 20) und der Vortragenden (etwa 150) liegt in den Händen der IMU bzw. des von ihr ernannten Programmkomitees. Vorsitzender dieses Komitees ist Phillip Griffiths vom Institute for Advanced Studies in Princeton, Deutschland ist mit zwei Mitgliedern im Komitee vertreten. Der Kongreß in Berlin dauert vom 18.-27. August 1998. Der erste Tag, der traditionell der Verleihung der Fields-Medaillen gewidmet ist, findet im Internationalen Congress Centrum statt. Für den Rest der Tagung übersiedeln wir dann an die TU Berlin.

\section{Stipendienprogramme}

Es ist der IMU seit langem ein Anliegen, die Teilnahme von Mathematikern aus den Ländern der Dritten Welt zu fördern, und wir haben uns diesem Vorhaben natürlich gerne angeschlossen. Die Absprache mit der IMU sieht vor, daß diese für die Reisemittel aufkommt und wir den Aufenthalt in Berlin finanzieren. Mit Hilfe öffentlicher Stellen hoffen wir, 100 bis 150 Stipendien vergeben zu können. Zusätzlich sind wir bemüht, durch großzügige Unterstützung möglichst viele Wissenschaftler aus Osteuropa nach Berlin einzuladen. Auch hier wurden mit den entsprechenden Stellen Kontakte aufgenommen. Es ist nur zu hoffen, daß beide Programme möglichst unbürokratisch abgewickelt werden können.

\section{Aktivitäten für die Öffentlichkeit \\ Der Internationale Kongreß bietet eine große und vielleicht einmalige Chance, das Wesen und die Be- deutung der Mathematik einer breiten Öffentlichkeit nahezubringen. Mit der Idee, den Kongreß durch ei- ne Reihe von außerordentlichen Veranstaltungen für}


das allgemeine Publikum zu öffnen, betreten wir Neuland -wir sind daher für alle Anregungen dankbar. In Berlin gibt es die Urania, die für solche Veranstaltungen eine lange Tradition hat. Es ist geplant, die Urania als Begegnungsort für die Dauer des Kongresses zu mieten und jeden Tag öffentliche Veranstaltungen anzubieten. Gedacht ist unter anderem an eine Reihe Mathematik und Musik, an eine Ausstellung über Mathematik in der Kunst, an eine Präsentation mathematischer Modelle (in Zusammenarbeit mit dem Deutschen Museum München), an Filme sowie mehrere Abendvorträge über Mathematik und ihre Beziehungen zu anderen Wissenschaften.

\section{Unterbringung in Berlin}

Ein schwieriges Problem, vor allem für Teilnehmer aus finanziell schwächer gestellten Ländern, stellt die Unterbringung dar. Die nicht ganz befriedigende Teilnehmerzahl in Zürich 1994 war sicherlich zum großen Teil auf die hohen Hotelkosten zurückzuführen. Da wir auf diese Kosten kaum Einfluß nehmen können, sind wir bemüht, in Berlin eine große Anzahl von preiswerten Pensionen und Privatquartieren anzubieten. Eine Sache hat uns in Zürich besonders gefallen, daß jeder Teilnehmer bei der Ankunft einen Ausweis zur Benützung aller öffentlicher Verkehrsmittel überreicht bekam. Wenn es die Finanzen erlauben, wollen wir diese Idee für Berlin übernehmen. Ansonsten sollte beim Rahmenprogramm in Berlin für jeden Geschmack etwas dabeisein -und ein paar Extras werden uns auch noch einfallen.

\section{Tagungen rund um den ICM 98}

Eine zusätzliche Attraktion für die Besucher des Kongresses, insbesondere für jene, die von weit herkommen, stellen Fachtagungen unmittelbar vor oder nach dem Kongreß dar. Bisher sind über 20 Vorschläge zu solchen Tagungen eingegangen, deren Tagungsort natürlich keineswegs auf Deutschland beschränkt sein muß. Wer eine Tagung veranstalten will, möge sich an den Koordinator, Herrn Frey (Uni Essen), wenden. Alle Tagungen werden in den Aussendungen und im offiziellen Programm bekanntgegeben.

\section{Informationen über den Kongreß}

Alles Wissenswerte über den ICM 98 können Sie im Internet unter der Adresse http://elib.zib-berlin.de/icm98 nachlesen. Dort werden die Informationen ständig auf den neuesten Stand gebracht. Außerdem finden Sie dort Informationen über die IMU, über die bisherigen Fields Medaillen Preisträger, das wissenschaftliche Programm, die bisher geplanten Rahmentagungen und anderes mehr. Und last but not least ist eine vorläufige Anmeldung über diese Adresse möglich. Mehrere hundert Mathematiker aus dem In- und Ausland haben davon bereits Gebrauch gemacht.

Zuletzt noch zwei Bitten: Werden Sie Mitglied im VICM98, dem Verein zur Durchführung des ICM 98. Der Jahresbeitrag von DM 30,-- soll ebenfalls in Stipendienprogramme fließen. Eine Anmeldung für den Verein ist brieflich oder per e-mail an

Prof.Dr. Jürgen Sprekels, Weierstraß-Institut für Angewandte Analysis und Stochastik, Mohrenstr. 39, 10117 Berlin, e-mail: sprekels@wias-berlin.de zu richten. Den Beitrag bitte unter dem Kennwort VICM98 e.V. bei der Berliner Bank, BLZ 100200 00, Konto Nr. 4388632900 einzahlen.

Und schließlich bitten wir Sie, Ihre Kollegen über den Kongreß zu informieren und über die Möglichkeit der elektronischen Anmeldung. Wir alle möchten, daß der ICM 98 ein Fest für die Mathematik wird, und dazu gehören alle, die sich für unsere Wissenschaft interessieren

Adresse des Autors:

Martin Aigner

Institut für Mathematik II

Freie Universität Berlin

Arnimallee 3, 14195 Berlin 